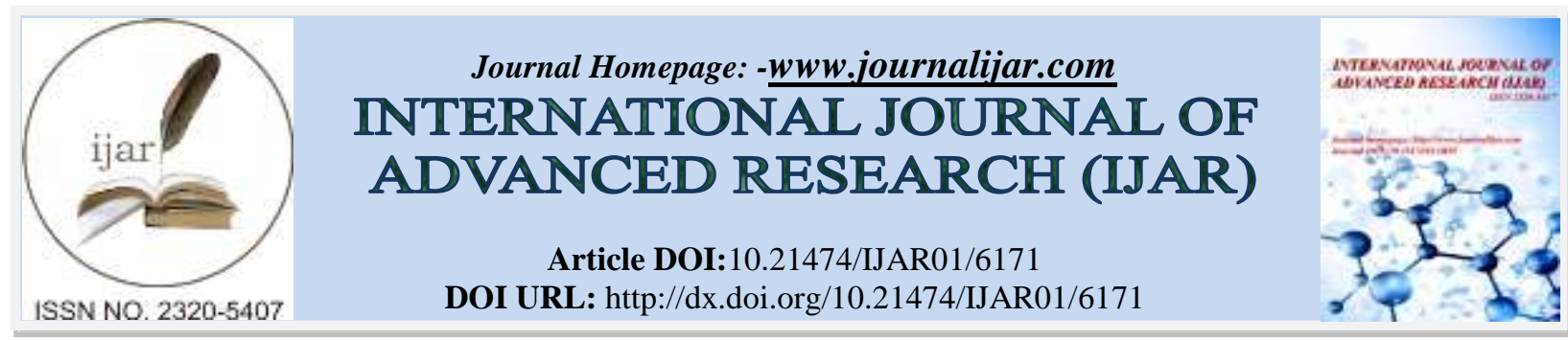

RESEARCH ARTICLE

\title{
BREAST TUBERCULOSIS PRESENTING AS A LUMP: AN UNCOMMON IDENTITY -A REPORT OF THREE CASES.
}

Dr. Meenu Gill ${ }^{1}$, Dr. Promil Jain ${ }^{2}$, Dr. Deepshikha ${ }^{3}$, Dr. Sumiti Gupta ${ }^{4}$ and Dr. Rajiv Sen ${ }^{5}$

1. Professor, Department of Pathology, Pt. B.D. Sharma PGIMS Rohtak.

2. Assistant Professor, Department of Pathology, Pt. B.D. Sharma PGIMS Rohtak.

3. Post-Graduate Resident, Department of Pathology, Pt. B.D. Sharma PGIMS Rohtak.

4. Professor, Department of Pathology, Pt. B.D. Sharma PGIMS Rohtak.

5. Senior Professor and Head, Department of Pathology, Pt. B.D. Sharma PGIMS Rohtak.

\section{Manuscript Info}

Manuscript History

Received: 25 October 2017

Final Accepted: 27 November 2017

Published: December 2017

Keywords:-

Breast tuberculosis, lump breast, ZiehlNeelsen.

\section{Abstract}

Breast tuberculosis is a rare manifestation of extra-pulmonary localization of the disease and accounts for only 3-4\% of breast conditions in underdeveloped regions of Asia and Africa with most of the cases seen in women in their reproductive age-groups. We encountered three such cases presenting as breast lump in a 16 year old, 26 year old and 70 year old female respectively. The overlying skin was normal in all the three cases and all of them came positive for Ziehl-Neelsen staining for acid-fast bacilli and were reported as tuberculous lymphadenitis involving intra-mammary lymph node, tuberculous mastitis and tuberculous inflammation respectively. The lump can sometimes be mistaken for breast cancer and pyogenic breast abscess. Fine needle aspiration cytology may not always detect the responsible pathogen but the presence of epithelioid cell granulomas and necrosis gives a clue to the establishment of a definitive diagnosis in upto $73 \%$ of the cases. A trial of anti-tubercular therapy with regular clinical assessment is warranted in all such cases.

Copy Right, IJAR, 2017,. All rights reserved.

\section{Introduction:-}

Breast tuberculosis is a rare manifestation of extra-pulmonary localization of tuberculosis and accounts for less than $0.1 \%$ of breast conditions in developed countries. However, it can reach up to 3-4\% in regions with high incidence of the disease in underdeveloped regions of Asia and Africa with most of the cases encountered in women in their reproductive age-groups. ${ }^{1}$ Although over one billion people are suffering from tuberculosis worldwide, breast tuberculosis is a relatively rare condition..$^{2}$ When the infection is seen affecting breast solely, the disease may be of primary etiology and when it results because of some other foci in the body, it is then termed as secondary tuberculosis of breast. This may have a varied presentation and may be confused with other entities and thus the clinician may confuse tuberculous mastitis with either carcinoma or breast abscess clinically as well as radiologically. ${ }^{3}$ Therefore, it remains a challenge for clinicians in diagnosing breast tuberculosis and requires a high degree of suspicion. Ultrasonography (USG) and mammography are unreliable diagnostic tools in differentiating breast tuberculosis from carcinoma because of the variable pattern of presentation of such lesions. Fine needle aspiration cytology (FNAC) plays a crucial role in the diagnosis of breast tuberculosis. Fine needle aspiration 
cytology may not always detect the responsible pathogen but the presence of epithelioid cell granulomas and necrosis gives a clue to establish a definitive diagnosis in up to $73 \%$ of cases.

\section{Case report:-}

We encountered three such cases. One of them was in a 16 year old female who presented with a painless lump in left breast since last one month. She gave a history of low grade fever on and off for the last 2 weeks. There were no other complaints like weight loss, loss of appetite or cough. She was unmarried and there was no positive family history of breast tuberculosis. USG showed multiple hypoechoic lymph nodes with areas of necrosis and maintained cortico-medullary differentiation. On local examination, overlying skin was normal and a non-tender, firm and mobile lump of size $1.5 \times 1.5 \mathrm{~cm}$ was felt in upper outer quadrant of the breast but no axillary nodes were palpable. Her systemic examination was unremarkable. Fine Needle Aspiration (FNA) was done and the smears prepared showed necrotizing granulomatous lymphadenitis (Fig. 1). Ziehl Neelsen (ZN) staining applied for acid fast bacilli came positive and a diagnosis of tuberculous lymphadenitis involving intra-mammary lymph node was made.

The second case was in a 26 year old female who presented with a painful lump in left breast since last 10 days which was progressively increasing in size. There was history of fever but no positive history of trauma, weight loss or loss of appetite. She was married and there was no positive family history of tuberculosis. On USG, left breast showed ectatic ducts in retroareolar region with echogenic contents which showed no flow on colour-doppler and tentative diagnosis of ductal ectasia was made. On local examination, the overlying skin was normal and a tender, firm lump measuring $4 \times 4 \mathrm{~cm}$ was felt in retroareolar region of left breast. Her systemic examination was unremarkable. FNA was done and the smears prepared showed groups and clusters of benign duct epithelial cells and histiocytes assuming epithelioid cell appearance in a background of neutrophils and RBCs. ZN staining applied for acid fast bacilli came positive and a diagnosis of tuberculous mastitis was made.

The third case was of a 70 year old female who presented with a swelling in her left breast for the last one and a half year. She gave history of on and off fever and loss of appetite but there was no history of cough or weight loss. Also there was no family history of tuberculosis. On local examination, overlying skin was unremarkable and an illdefined nodular mass of size $4 \times 4 \mathrm{~cm}$ was felt in upper inner quadrant of the breast. FNA done from the lump showed numerous intact and degenerated neutrophils, lymphocytes, macrophages and occasional histiocytes in background of necrotic material and RBCs. Also many small and large groups of degenerated apparently benign ductal cells were also seen. (Fig. 2) ZN staining for AFB was positive and this led to a diagnosis of tuberculous inflammation. (Fig. 3)

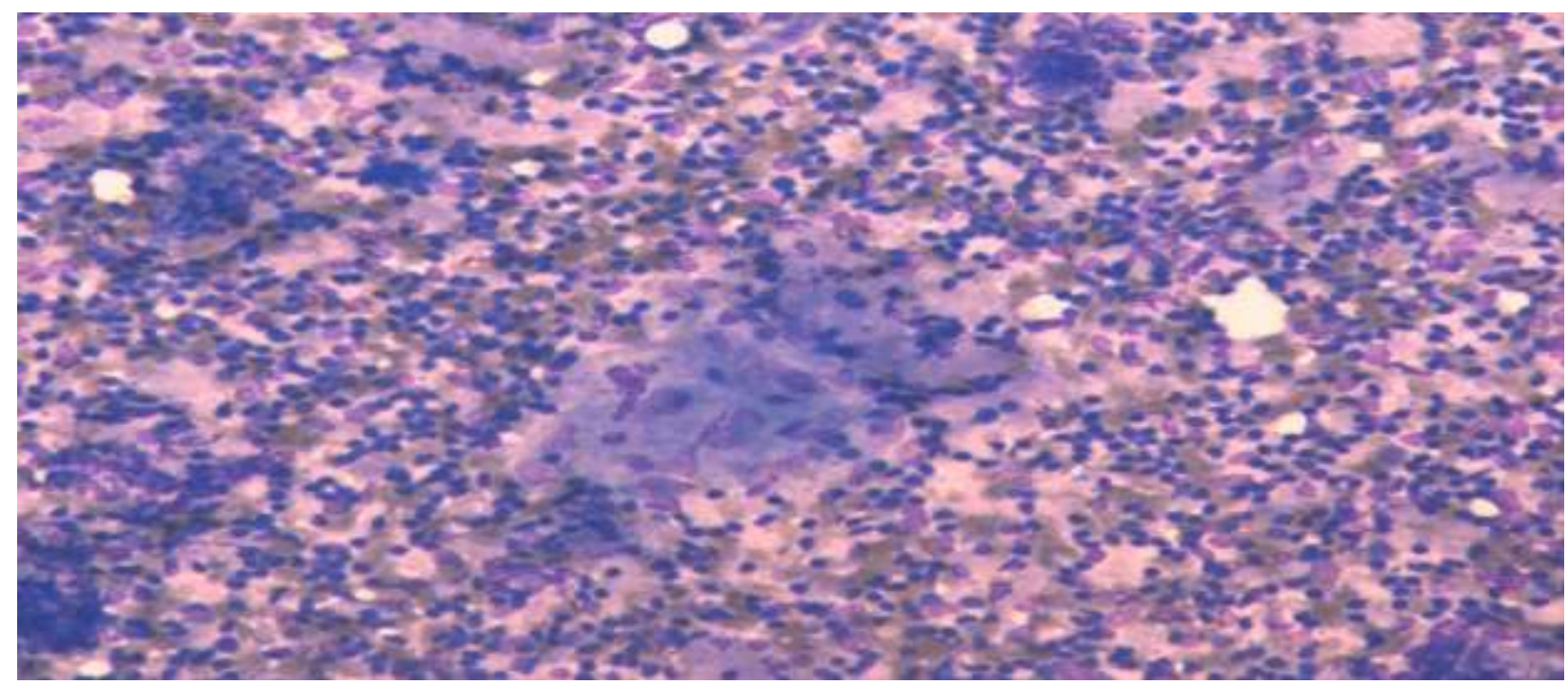

Figure 1:- Photomicrograph showing granuloma formation. (Leishman, 200x) 


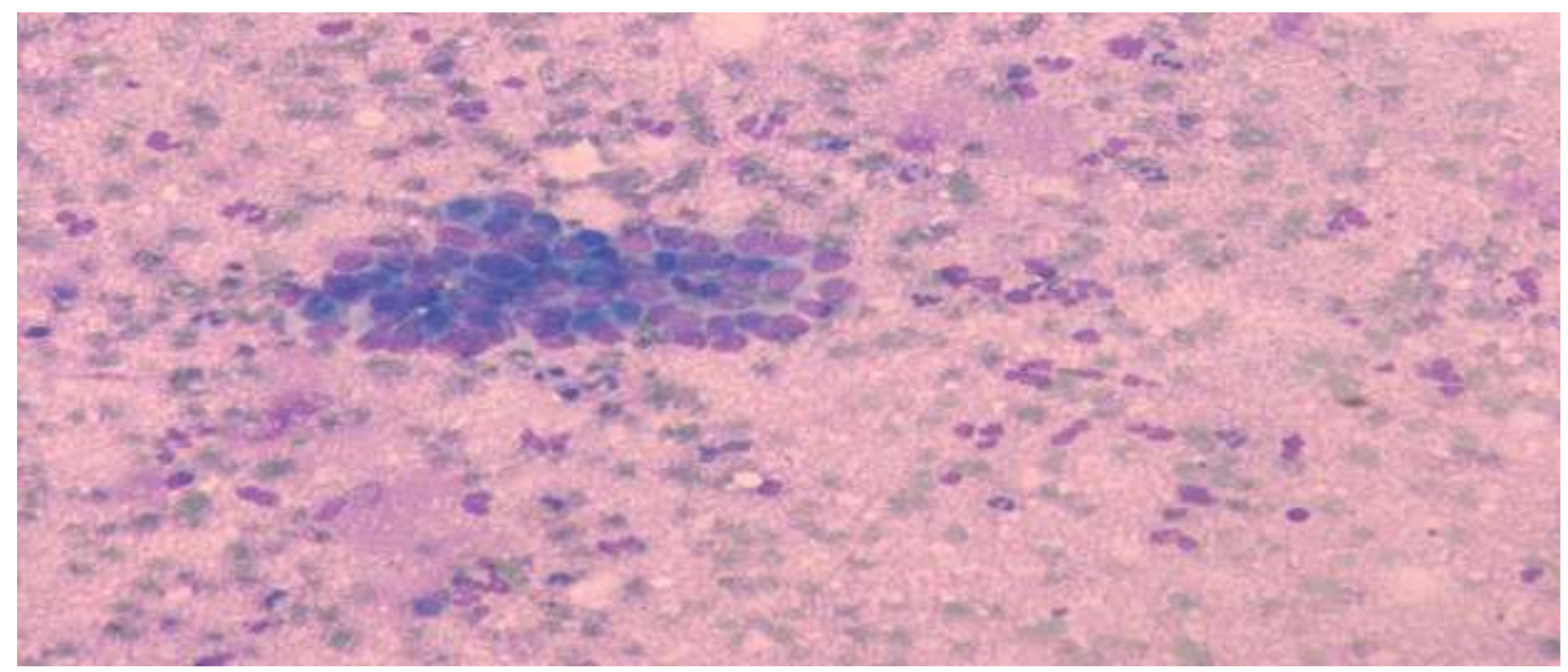

Figure 2:- Photomicrograph showing benign ductal epithelial cell sheet, inflammatory infiltrate and necrotic background. (Leishman, 200x)

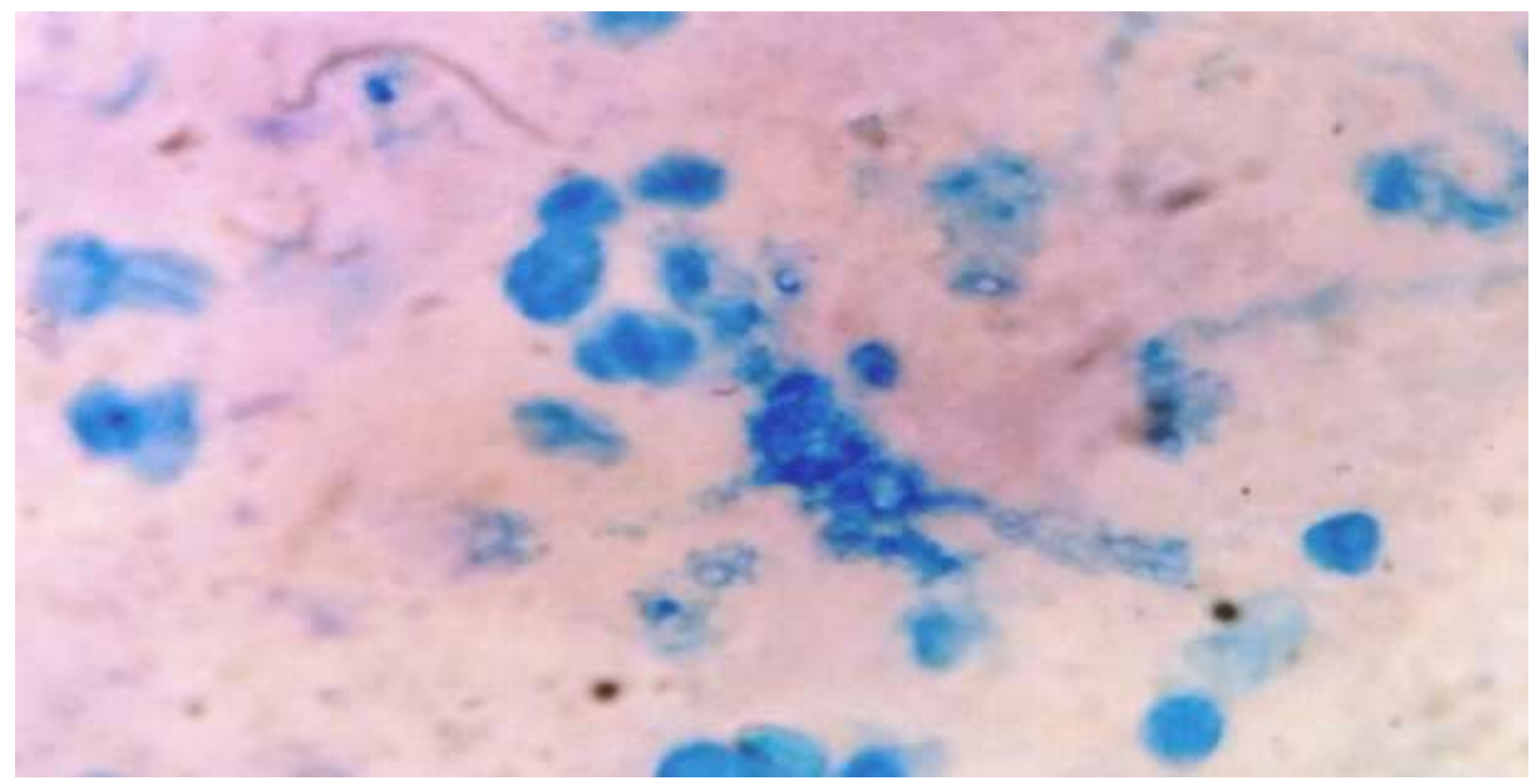

Figure 3:- Photomicrograph showing acid fast bacilli (ZN staining, oil immersion, 1000x).

\section{Discussion:-}

Tuberculosis of the breast is an uncommon identity, mostly because organs or tissues like the breast, skeletal muscle and spleen are more resistant to infection, making the survival as well as multiplication of the tubercle bacilli difficult. High incidence of breast tuberculosis is presumed in India despite only few hundred cases of breast tuberculosis reported, probably due to lack of awareness of manifestation of disease or misdiagnosis. ${ }^{4}$ There can be unilateral or bilateral involvement of breast but there are only few reports which have documented bilateral breast tuberculosis in India. ${ }^{5}$ The risk factors which predispose to breast tuberculosis include multiparity, trauma during lactation phase, previous history of suppurative mastitis and immunocompromised states like AIDS. Infection can be acquired through various routes which include haematogenous, lymphatic, direct inoculation and ductal infection. Breast tuberculosis can present either as a lump, ulcer or breast abscess with or without discharging sinuses. The lump can sometimes mimic breast carcinoma, being hard, with irregular border, fixed to either the skin, muscle or even to the chest wall. Chest X-ray, Mantoux skin test, ESR, USG can provide additive information in arriving at a diagnosis. The extra-mammary extent of the disease can be demonstrated by the use of Magnetic Resonance 
Imaging. The gold standard for the diagnosis of breast tuberculosis is detection of M. tuberculosis by Ziehl Neelsen staining or by culture. Fine needle aspiration cytology may not always detect the responsible pathogen but the presence of epithelioid cell granulomas and necrosis gives a clue to arrive at a definitive diagnosis in up to $73 \%$ of cases. Fine needle aspiration cytology and bacteriological studies provide us with a definitive diagnosis which can further be complemented with a biopsy. Antitubercular therapy (ATT) with or without minimal surgical intervention forms the mainstay of treatment. Surgical intervention is required for aspiration of abscesses and excision of sinuses and masses. In resistant cases, simple mastectomy can be performed. ${ }^{6}$

\section{Conclusion:-}

The significance of breast tuberculosis is due to its rare entity and mistaken identity with breast cancer and pyogenic breast abscess. If there is a high clinical suspicion of $\mathrm{TB}$, a trial of anti-tubercular therapy with regular clinical assessment may be given.

\section{References:-}

1. De Sousa R, Patil R. Breast tuberculosis or granulomatous mastitis: A diagnostic dilemma. Ann Trop Med Public Health. 2011;4:122-5.

2. Singal R, Bala J, Gupta S, Goyal S, Mahajan NC, Chawla A. Primary breast tuberculosis presenting as a lump: a rare modern disease. Ann Med Health Sci Res. 2013; 3(1):110-12.

3. Madhusudhan KS, Gamanagatti S. Primary breast tuberculosis masquerading as carcinoma. Singapore Med J. 2008; 49(1):3-5.

4. Banerjee SN, Ananthakrishnan N, Mehta RB, Parkash S. Tuberculous mastitis: a continuing problem. World J Surg. 1987; 11(1):105-9.

5. Bedi U, Bedi R. Bilateral breast tuberculosis. Ind J Tuberc. 2001; 48:215-7.

6. Tewari M, Shukla HS. Breast tuberculosis: diagnosis, clinical features \& management. Ind J Med Res. 2005; 122(2):103-10. 